Research Article

\title{
Further Stability Analysis on Neutral Systems with Actuator Saturation and Time-Delays
}

\author{
Xinghua Liu \\ Department of Automation, School of Information Science and Technology, University of Science and Technology of China, \\ Anhui 230027, China \\ Correspondence should be addressed to Xinghua Liu; salxh@mail.ustc.edu.cn
}

Received 13 January 2014; Revised 3 April 2014; Accepted 7 April 2014; Published 4 May 2014

Academic Editor: Kartik Ariyur

Copyright (C) 2014 Xinghua Liu. This is an open access article distributed under the Creative Commons Attribution License, which permits unrestricted use, distribution, and reproduction in any medium, provided the original work is properly cited.

This paper is concerned with the asymptotic stability analysis for a class of neutral systems with time-delay and saturating actuators, which is further to reduce the conservatism of neutral system. Based on the model transformation and the delay-dividing approach, a new type of augmented Lyapunov functional is constructed, which has fully exploited the information on the lower bound of the delay. Then the delay-dependent conditions for asymptotic stability are derived by applying some integral inequalities and Lyapunov theory. Finally, numerical examples are provided to show that the proposed results significantly improve the allowed upper bounds of the delay size over some existing ones in the literature.

\section{Introduction}

As is well known, time-delay is often encountered in various mechanical and engineering systems, such as aircraft stabilization, manual control, microwave oscillator, models of lasers, rolling mill systems, and neural network. Since it is shown that the existence of delays may result in instability, oscillations, or poor performances, the problem of stability analysis of time-delay systems has received considerable attention over the decades. Various approaches have been proposed in the literature to obtain stability criteria for delay systems, such as the Lyapunov technique, characteristic equation method, and state solution approaches. In the real world, there are many practical systems containing timedelay of neutral type, that is, the derivative of the past state; we called them neutral systems. Such systems can be found in population ecology [1], processes including steam or water pipes, heat exchanges [2], and other areas [3]. Existing stability criteria of delay systems can be classified into two categories, that is, delay-independent ones and delay-dependent ones. It is generally recognized that delay-independent ones are more conservative than the delay-dependent ones. So, in the literature of these years, the attention was mainly focused in giving delay-dependent stability conditions for neutral systems; see, for example, [4-17] and references therein for more details. However, there is some room for further investigation to reduce the conservatism of neutral systems by novel method.

Besides delays, the saturated controller is apt to cause instability as well. In various practical systems, actuator saturation and time-delays are often observed together in control systems and they are frequently a source of instability. Therefore, much attention has been paid to derive some delay-dependent stability conditions for the time-delay systems with actuator saturation. Based on the LyapunovKrasovskii functional, the sufficient conditions have been discussed in [18-23] and the references therein. The controller is constructed in terms of the solution to a set of linear matrix inequalities by employing descriptor model transformation of the systems in [21]. The Lyapunov-Krasovskii functional is employed to investigate the problem of delaydependent robust stabilization of uncertain neutral systems with saturating actuators in [20], just to name a few. However, to the author's knowledge, it seems that the stability and control synthesis problem of neutral systems in the presence of actuator saturation has not been fully investigated and it is very challenging.

This paper is concerned with the asymptotic stability problem of neutral systems with actuator saturation and the objective is to propose some new delay-dependent 
conditions, which are less conservative than some existing ones in the literature. The design approach is dependent on the discrete time-delay and neutral time-delay. Applying the delay-dividing approach, the asymptotic stability conditions are formulated as linear matrix inequalities (LMIs) by the model transformation and Lyapunov method. Facing the uncertain case, we obtain the robust asymptotic stability conditions under such hypothesis condition that the system has the norm bounded uncertainties. At last, examples are provided to demonstrate the reduced conservativeness of the proposed criteria by numerically comparing with those reported previously in the literature. The main contributions of this paper can be summarized as follows: (i) model transformation is considered in this paper, but we employ a new stability condition of the transformation operator $\mathscr{L}$ to reduce the conservatism; (ii) the bound of the delay is fully utilized in this paper, that is, improved bounding technique or delay-dividing approach is used to reduce the conservatism; (iii) the constructed Lyapunov functional is novel and very effective in the reduction of conservatism, which has not appeared in the context of neutral systems with time-delay and actuator saturation before; (iv) numerical examples and simulations are provided to verify that the proposed result in this paper is less conservative than some existing ones.

The remainder of this paper is organized as follows: Section 2 contains the problem statement and preliminaries; Section 3 presents the main results; Section 4 provides some numerical examples to verify the effectiveness of the results; Section 5 draws a brief conclusion.

1.1. Notations. In this paper, $\star$ denotes the elements below the main diagonal of a symmetric block matrix, $\mathbb{R}^{n}$ denotes the $n$ dimensional Euclidean space, and $\mathbb{R}^{m \times n}$ is for the set of all real $m \times n$ matrices. $I$ denotes the identity matrix with proper dimensions. $C_{0}$ is the set of all continuous functions from $[-\tau, 0]$ to $\mathbb{R}^{n}$ and $\tau$ is constant neutral time-delay. $A^{T}$ is the transpose of matrix $A .|A|=\left[\left|a_{i j}\right|\right]$, with $A=\left[a_{i j}\right]$. For real symmetric matrices $X$ and $Y$, the notation $X \geq Y$ (resp., $X>Y$ ) means that the matrix $X-Y$ is positive semidefinite (resp., positive definite). $\lambda_{\max (\min )}(A)$ means the eigenvalue of matrix $A$ with maximum (minimum) real part. $\|v\|$ means the Euclidean norm of vector $v,\|v\|=\left(v^{T} v\right)^{1 / 2}$, while $\|A\|$ is spectral norm of matrix $A,\|A\|=\left[\lambda_{\max }\left(A^{T} A\right)\right]^{1 / 2}$. $\operatorname{diag}\{\cdots\}$ denotes a block-diagonal matrix decided by the corresponding elements in the brace. In addition, if not explicitly stated, matrices are assumed to have compatible dimensions.

\section{Problem Statement and Preliminaries}

In this paper, the investigated neutral systems with time-delay and actuator saturation are described by the following:

$$
\begin{aligned}
\dot{x}(t)-D \dot{x}(t-\tau) & =A x(t)+B x(t-h)+C S a t(u(t)), \\
x(s) & =\phi(s), \quad s \in[-\rho, 0]
\end{aligned}
$$

where $x(t) \in \mathbb{R}^{n}$ is the system state and $u(t)$ is the control input. $h>0$ is a constant discrete time-delay and $\tau>0$ is a constant neutral time-delay. $A, B, C$, and $D$ are known real constant parameter matrices of appropriate dimensions. To apply the Lyapunov stability theorem [2] we assume that $\|D\|<1$. The initial condition $\phi(s)$ is a continuously differentiable vector-valued function. The continuous norm of $\phi(s)$ is defined as

$$
\|\phi\|_{c}=\max _{s \in[-\rho, 0]}|\phi(s)|, \quad \rho=\max \{\tau, h\} .
$$

Linear state feedback is described by the following:

$$
u(t)=K x(t)
$$

the linear state feedback gain $K$ is denoted as

$$
K=\left[K_{1}^{T}, K_{2}^{T}, \ldots, K_{m}^{T}\right]^{T}
$$

$K_{i}(i=1,2, \ldots, m)$ is an $n$-dimension row vector.

The notation Sat(.) is to denote the standard saturation function (Figure 1 ) defined as $u=\left[u_{1}, u_{2}, \ldots, u_{m}\right]$ :

$$
\begin{gathered}
\text { Sat }(u)=\left[\operatorname{Sat}\left(u_{1}\right), \operatorname{Sat}\left(u_{2}\right), \ldots, \text { Sat }\left(u_{m}\right)\right]^{T}, \\
\text { Sat }\left(u_{i}\right)= \begin{cases}u_{i}^{+} & \text {if } u_{i}>u_{i}^{+}, \\
u_{i} & \text { otherwise, } \\
-u_{i}^{+} & \text {if } u_{i}<-u_{i}^{+} .\end{cases}
\end{gathered}
$$

Here we have slightly abused the notation by using Sat to denote both a scalar valued and a vector valued function. We have also assumed a unity saturation level for the saturation function without loss of generality. Let us decompose the saturation nonlinear function as the sum of a linear part and another nonlinear part.

Definition 1. Nonlinear part $D z(u(t))=u(t)-\operatorname{Sat}(u(t))$, where $D z(u(t))=\left[d z\left(u_{1}\right), d z\left(u_{2}\right), \ldots, d z\left(u_{m}\right)\right]^{T}$

$$
d z\left(u_{i}\right)= \begin{cases}u_{i}-u_{i}^{+} & \text {if } u_{i}>u_{i}^{+} \\ 0 & \text { otherwise } \\ u_{i}+u_{i}^{+} & \text {if } u_{i}<-u_{i}^{+}\end{cases}
$$

Making use of nonlinear part $D z(u(t))$, we rewrite the saturated system in the following form:

$$
\dot{x}(t)-D \dot{x}(t-\tau)=A_{c} x(t)+B x(t-h)-C D z(K x(t)),
$$

where $A_{c}=A+C K$.

The purpose of this paper is to investigate the sensitivity of stability to variations in the parameters and in the delays. Notice that the stability of a neutral system requires a necessary condition to guarantee the stability of the associated difference operator [24]. Under normal circumstances, we deal with the difference operator given by

$$
\mathscr{D}(x(t))=x(t)-D x(t-\tau) .
$$




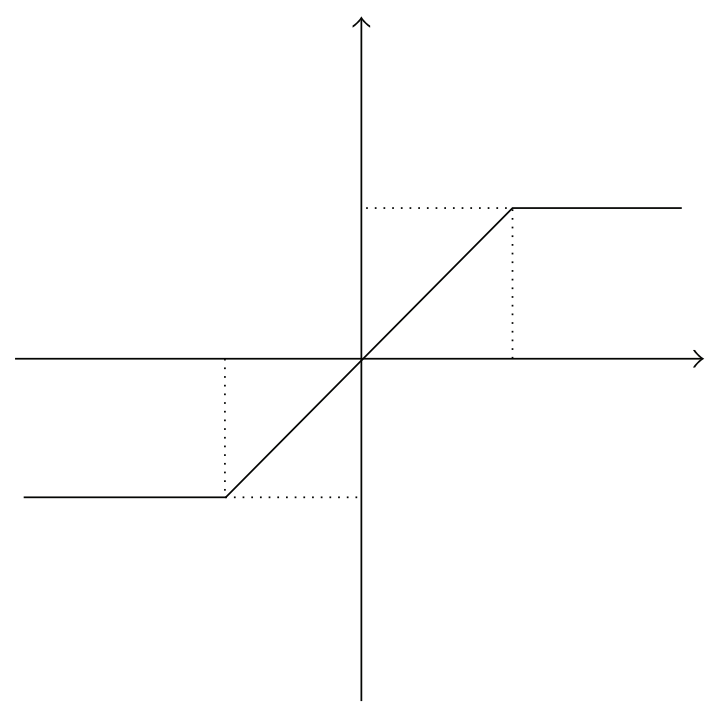

FIGURE 1: A saturation function $\operatorname{Sat}\left(u_{i}(t)\right)$.

Then the stability of operator $\mathscr{D}$ is ensured if $\|D\|<1$. However, in this paper we introduce a model transformation, which imply the stability of the original model and can be studied by means of Lyapunov-Krasovskii functional.

Utilizing the model transformation, the neutral system (7) can be written as

$$
\frac{d}{d t}[\mathscr{L}(x(t))]=\widehat{A}_{c} x(t)-C D z(K x)
$$

where $\widehat{A}_{c}=A_{c}+B=A+B+C K, \mathscr{L}(x(t))=x(t)-D x(t-$ $\tau)+B \int_{t-h}^{t} x(s) d s$.

Remark 2. The proposed model transformation which uses Leibnitz's rule does not induce any additional dynamics in the characteristic equation for the system without uncertainties (see more details $[25,26]$ ).

It is not difficult to verify that every solution of the neutral system (7) is also solution of (9); then the stability of (9) implies the stability of (7); see [27]. However, the stability of operator $\mathscr{L}$ is required, and it is more difficult to tackle than the untransformed system. In order to give the less conservative stability condition, we introduce Definition 3 and Lemma 4.

Definition 3 (see [24]). The operator $\mathscr{L}: C_{0} \rightarrow \mathbb{R}^{n}$ is said to be stable if the zero solution of the homogeneous difference equation

$$
\begin{aligned}
& \mathscr{L}(x(t))=0, \quad t \geq 0, \\
& x_{0}=\psi \in\left\{\varphi \in C_{0}: \mathscr{L} \varphi=0\right\}
\end{aligned}
$$

is uniformly asymptotically stable. The stability of operator $\mathscr{L}$ is necessary for the stability of neutral system (1) with (3), which is always satisfied when $\|h|B|+D\|<1$.
Lemma 4 (see [28]). For given positive scalars $\alpha_{1}$ and $\alpha_{2}$, the operator $\mathscr{L}: C_{0} \rightarrow \mathbb{R}^{n}$ defined by

$$
\mathscr{L}(x(t))=x(t)+B \int_{t-h}^{t} x(s) d s-D x(t-\tau)
$$

is stable if there exists a positive-definite matrix $M$ such that

(i) $\alpha_{1}+\alpha_{2}<1$,

(ii) $\left[\begin{array}{cc}D^{T} M D-\alpha_{1} M & h D^{T} M B \\ \star & h^{2} B^{T} M B-\alpha_{2} M\end{array}\right]<0$.

Remark 5. As said in [28], the above criterion (12) is less conservative than the well-known criterion for the stability of the operator $\mathscr{L}$ given in [24]. So the criterion (12) is exploited in this paper to reduce the conservatism of the neutral system with time-delay and actuator saturation.

Then we give the following two lemmas before proceeding main results.

Lemma 6 (see [29]). Let $U \in R^{n \times n}$ and $V \in R^{n \times n}$, and let $x \in R^{n}$. Then we have

$$
\begin{gathered}
x^{T} U V x \leq\|U V\| x^{T} x \leq\|U\|\|V\| x^{T} x, \\
2 x^{T} U V x \leq x^{T}\left(\frac{1}{\gamma} U U^{T}+\gamma V^{T} V\right) x, \quad \forall \gamma>0 .
\end{gathered}
$$

Considering a matrix $G \in \mathbb{R}^{m \times n}$ and defining the following polyhedral set

$$
\mathcal{S} \triangleq\left\{x \in \mathbb{R}^{n} ;\left|\left(K_{i}-G_{i}\right) x\right| \leq u_{i}^{+}, i=1,2, \ldots, m\right\},
$$

where $G_{i}$ is the row vector of matrix $G$, the following Lemma 7 concerning the nonlinearity $D z(K x(t))$ can be stated.

Lemma 7 (see [30]). Consider the function $D z(K x)$ defined in (6). If $x \in \mathcal{S}$ then the relation

$$
D z^{T}(K x) \Lambda[D z(K x)-G x] \leq 0
$$

is verified for any diagonal and positive definite matrix $\Lambda \in$ $\mathbb{R}^{m \times m}$.

\section{Main Results}

In this section, we present the stability conditions which guarantee the asymptotic stability of the neutral system with time-delay and actuator saturation. Firstly, we deduce the sufficient asymptotic stability condition for (1) and (3). Then we manage to extend the sufficient conditions to the uncertain case.

\subsection{Asymptotic Stability for Certain Neutral System}

Theorem 8. Given scalars $\alpha_{1}$ and $\alpha_{2}$, a matrix $G$, and a diagonal matrix $\Lambda$ of appropriate dimensions, the neutral system with time-delay and actuator saturation which can 
be described by (1) and (3) is asymptotically stable if there exists a positive definite matrix $M$ satisfying (12) and there exist $P>0, Q=\left[Q_{i j}\right]_{2 \times 2}, Q_{i j}>0, R=\left[R_{i j}\right]_{2 \times 2}, R_{i j}>0, T_{i}>0$, $(i=1,2,3,4,5,6), \widetilde{T}_{i}>0,(i=3,4)$ such that the following symmetric linear matrix inequality holds:

$$
\Phi=\left[\begin{array}{cccccccc}
\Phi_{11} & \Phi_{12} & 0 & \Phi_{14} & \Phi_{15} & \Phi_{16} & 0 & \Phi_{18} \\
\star & \Phi_{22} & \Phi_{23} & 0 & 0 & 0 & 0 & 0 \\
\star & \star & \Phi_{33} & 0 & 0 & 0 & 0 & 0 \\
\star & \star & \star & \Phi_{44} & \Phi_{45} & 0 & 0 & 0 \\
\star & \star & \star & \star & \Phi_{55} & 0 & 0 & \Phi_{58} \\
\star & \star & \star & \star & \star & \Phi_{66} & 0 & \Phi_{68} \\
\star & \star & \star & \star & \star & \star & \Phi_{77} & 0 \\
\star & \star & \star & \star & \star & \star & \star & \Phi_{88}
\end{array}\right]<0,
$$

where $\widehat{G}=\Lambda G, \widetilde{T_{3}}=T_{3} T_{5}^{-1} T_{3}$, and $\widetilde{T_{4}}=T_{4} T_{6}^{-1} T_{4}$

$$
\begin{aligned}
\Phi_{11}= & \widehat{A}_{c}^{T} P+P \widehat{A}_{c}+Q_{11}+R_{11}+T_{1}+T_{2} \\
& +\frac{h^{2}}{2}\left(\widetilde{T_{3}}+T_{5}\right)+\frac{\tau^{2}}{2}\left(\widetilde{T_{4}}+T_{6}\right), \\
\Phi_{22}= & Q_{22}-Q_{11}, \\
\Phi_{33}= & -Q_{22}-T_{1}, \\
\Phi_{44}= & R_{22}-R_{11}, \\
\Phi_{55}= & -R_{22}-T_{2}, \\
\Phi_{66}= & -T_{3}, \\
\Phi_{77}= & -T_{4}, \\
\Phi_{88}= & -2 \Lambda, \\
\Phi_{12}= & Q_{12}, \\
\Phi_{14}= & R_{12} \\
\Phi_{15}= & -\widehat{A}_{c}^{T} P D \\
\Phi_{16}= & \widehat{A}_{c}^{T} P B \\
\Phi_{18}= & -P C+\widehat{G}, \\
\Phi_{23}= & -Q_{12}, \\
\Phi_{45}= & -R_{12}, \\
\Phi_{58}= & D^{T} P C \\
\Phi_{68}= & -B^{T} P C . \\
&
\end{aligned}
$$

Proof. Define a legitimate Lyapunov functional candidate as

$$
\begin{aligned}
V(x(t))= & V_{1}(x(t))+V_{2}(x(t))+V_{3}(x(t)) \\
& +V_{4}(x(t))+V_{5}(x(t))+V_{6}(x(t)),
\end{aligned}
$$

where

$$
\begin{aligned}
& V_{1}(x(t))=\mathscr{L}^{T}(x(t)) P \mathscr{L}(x(t)), \\
& V_{2}(x(t))=\int_{t-(h / 2)}^{t}\left[x\left(s-\frac{h}{2}\right)\right]^{x(s)}\left[\begin{array}{cc}
Q_{11} & Q_{12} \\
\star & Q_{22}
\end{array}\right] \\
& \times\left[x\left(s-\frac{h}{2}\right)\right] d s \\
& +\int_{t-(\tau / 2)}^{t}\left[x\left(s-\frac{\tau}{2}\right)\right]^{T}\left[\begin{array}{cc}
R_{11} & R_{12} \\
\star & R_{22}
\end{array}\right] \\
& \times\left[\begin{array}{c}
x(s) \\
x\left(s-\frac{\tau}{2}\right)
\end{array}\right] d s \\
& V_{3}(x(t))=\int_{t-h}^{t} x^{T}(s) T_{1} x(s) d s+\int_{t-\tau}^{t} x^{T}(s) T_{2} x(s) d s, \\
& V_{4}(x(t))=\int_{t-h}^{t}\left[\int_{s}^{t} x^{T}\left(s_{1}\right) d s_{1}\right] T_{3}\left[\int_{s}^{t} x\left(s_{2}\right) d s_{2}\right] d s, \\
& V_{5}(x(t))=\int_{t-\tau}^{t}\left[\int_{s}^{t} x^{T}\left(s_{1}\right) d s_{1}\right] T_{4}\left[\int_{s}^{t} x\left(s_{2}\right) d s_{2}\right] d s \text {, } \\
& V_{6}(x(t))=\frac{1}{2} \int_{t-h}^{t}(s-t+h)^{2} x^{T}(s) T_{5} x(s) d s \\
& +\frac{1}{2} \int_{t-\tau}^{t}(s-t+\tau)^{2} x^{T}(s) T_{6} x(s) d s,
\end{aligned}
$$

where $\mathscr{L}(x(t))$ has been defined in (9), $P>0, Q=\left[Q_{i j}\right]_{2 \times 2}$, $Q_{i j}>0, R=\left[R_{i j}\right]_{2 \times 2}, R_{i j}>0$, and $T_{i}>0, i=1,2,3,4,5,6$.

We solve the time derivative of $V(x(t))$ along the trajectories of the closed-loop system (9) and obtain the following:

$$
\begin{aligned}
\dot{V}(x(t))= & \dot{V}_{1}(x(t))+\dot{V}_{2}(x(t))+\dot{V}_{3}(x(t)) \\
& +\dot{V}_{4}(x(t))+\dot{V}_{5}(x(t))+\dot{V}_{6}(x(t)) .
\end{aligned}
$$

Given that $x(t) \in \mathcal{S}$, which has been discussed in [21]. From Lemma 7, it follows that

$$
\begin{aligned}
\dot{V}(x(t)) \leq & \dot{V}_{1}(x(t))+\dot{V}_{2}(x(t))+\dot{V}_{3}(x(t))+\dot{V}_{4}(x(t)) \\
& +\dot{V}_{5}(x(t))+\dot{V}_{6}(x(t)) \\
& -2 D z^{T}(K x) \Lambda[D z(K x)-G x] .
\end{aligned}
$$




$$
\begin{aligned}
& \dot{V}_{1}(x(t))=2\left\{x^{T}(t)-x^{T}(t-\tau) D^{T}\right. \\
& \left.+\left[\int_{t-h}^{t} x^{T}(s) d s\right] B^{T}\right\} \\
& \times P\left[\widehat{A}_{c} x(t)-C D z(K x)\right] \\
& =2 x^{T} P \widehat{A}_{c} x-2 x^{T} P C D z(K x) \\
& -2 x^{T} \widehat{A}_{c}^{T} P D x(t-\tau) \\
& +2 x^{T}(t-\tau) D^{T} P C D z(K x) \\
& +2 x^{T}(t) \widehat{A}_{c}^{T} P B\left[\int_{t-h}^{t} x(s) d s\right] \\
& -2\left[\int_{t-h}^{t} x^{T}(s) d s\right] B^{T} P C D z(K x) \\
& =x^{T}\left[\widehat{A}_{c}^{T} P+P \widehat{A}_{c}\right] x \\
& +2 x^{T}(t-\tau) D^{T} P C D z(K x) \\
& +2 x^{T}(t) \widehat{A}_{c}^{T} P B\left[\int_{t-h}^{t} x(s) d s\right] \\
& -2 x^{T} P C D z(K x) \\
& -2 x^{T}(t) \widehat{A}_{c}^{T} P D x(t-\tau) \\
& -2\left[\int_{t-h}^{t} x^{T}(s) d s\right] B^{T} P C D z(K x), \\
& \dot{V}_{2}(x(t))=\left[\begin{array}{c}
x(t) \\
x\left(t-\frac{h}{2}\right)
\end{array}\right]^{T}\left[\begin{array}{cc}
Q_{11} & Q_{12} \\
\star & Q_{22}
\end{array}\right]\left[\begin{array}{c}
x(t) \\
x\left(t-\frac{h}{2}\right)
\end{array}\right] \\
& +\int_{t-h}^{t} x^{T}(t) T_{3}\left[\int_{s}^{t} x\left(s_{2}\right) d s_{2}\right] d s \\
& +\int_{t-h}^{t}\left[\int_{s}^{t} x\left(s_{2}\right) d s_{2}\right] T_{3} x(t) d s \\
& =-\left[\int_{t-h}^{t} x^{T}\left(s_{1}\right) d s_{1}\right] T_{3}\left[\int_{t-h}^{t} x\left(s_{2}\right) d s_{2}\right] \\
& +\int_{t-h}^{t}(s-t+h) x^{T}(t) T_{3} x(s) d s \\
& +\int_{t-h}^{t}(s-t+h) x^{T}(s) T_{3} x(t) d s \\
& =-\left[\int_{t-h}^{t} x^{T}\left(s_{1}\right) d s_{1}\right] T_{3}\left[\int_{t-h}^{t} x\left(s_{2}\right) d s_{2}\right] \\
& -\int_{t-h}^{t}(s-t+h)\left(T_{3} x(t)-T_{5} x(s)\right)^{T} T_{5}^{-1} \\
& \times\left(T_{3} x(t)-T_{5} x(s)\right) d s \\
& +\frac{h^{2}}{2} x^{T}(t) T_{3} T_{5}^{-1} T_{3} x(t) \\
& +\int_{t-h}^{t}(s-t+h) x^{T}(s) T_{5} x(s) d s \\
& \leq-\left[\int_{t-h}^{t} x^{T}\left(s_{1}\right) d s_{1}\right] T_{3}\left[\int_{t-h}^{t} x\left(s_{2}\right) d s_{2}\right] \\
& +\frac{h^{2}}{2} x^{T}(t) \widetilde{T_{3}} x(t) \\
& +\int_{t-h}^{t}(s-t+h) x^{T}(s) T_{5} x(s) d s,
\end{aligned}
$$$$
-\left[\begin{array}{c}
x\left(t-\frac{h}{2}\right) \\
x(t-h)
\end{array}\right]^{T}\left[\begin{array}{cc}
Q_{11} & Q_{12} \\
\star & Q_{22}
\end{array}\right]\left[\begin{array}{c}
x\left(t-\frac{h}{2}\right) \\
x(t-h)
\end{array}\right]
$$$$
+\left[\begin{array}{c}
x(t) \\
x\left(t-\frac{\tau}{2}\right)
\end{array}\right]^{T}\left[\begin{array}{cc}
R_{11} & R_{12} \\
\star & R_{22}
\end{array}\right]\left[\begin{array}{c}
x(t) \\
x\left(t-\frac{\tau}{2}\right)
\end{array}\right]
$$$$
-\left[\begin{array}{c}
x\left(t-\frac{\tau}{2}\right) \\
x(t-\tau)
\end{array}\right]^{T}\left[\begin{array}{cc}
R_{11} & R_{12} \\
\star & R_{22}
\end{array}\right]\left[\begin{array}{c}
x\left(t-\frac{\tau}{2}\right) \\
x(t-\tau)
\end{array}\right],
$$$$
\dot{V}_{3}(x(t))=x^{T}(t) T_{1} x(t)-x^{T}(t-h) T_{1} x(t-h)
$$$$
+x^{T}(t) T_{2} x(t)-x^{T}(t-\tau) T_{2} x(t-\tau),
$$$$
\dot{V}_{4}(x(t))=-\left[\int_{t-h}^{t} x^{T}\left(s_{1}\right) d s_{1}\right] T_{3}\left[\int_{t-h}^{t} x\left(s_{2}\right) d s_{2}\right]
$$

where $\widetilde{T_{3}}>0$, since $\widetilde{T_{3}}=T_{3} T_{5}^{-1} T_{3}$.

Similarly we have

$$
\begin{aligned}
\dot{V}_{5}(x(t)) \leq & -\left[\int_{t-\tau}^{t} x^{T}\left(s_{1}\right) d s_{1}\right] T_{4}\left[\int_{t-\tau}^{t} x\left(s_{2}\right) d s_{2}\right] \\
& +\frac{\tau^{2}}{2} x^{T}(t) \widetilde{T_{4}} x(t) \\
& +\int_{t-\tau}^{t}(s-t+\tau) x^{T}(s) T_{6} x(s) d s
\end{aligned}
$$

where $\widetilde{T_{4}}>0$, since $\widetilde{T_{4}}=T_{4} T_{6}^{-1} T_{4}$. Considering

$$
\begin{aligned}
\dot{V}_{6}(x(t))= & \frac{1}{2} h^{2} x^{T}(t) T_{5} x(t) \\
& -\int_{t-h}^{t}(s-t+h) x^{T}(s) T_{5} x(s) d s
\end{aligned}
$$




$$
\begin{aligned}
& +\frac{1}{2} \tau^{2} x^{T}(t) T_{6} x(t) \\
& -\int_{t-\tau}^{t}(s-t+\tau) x^{T}(s) T_{6} x(s) d s .
\end{aligned}
$$

Substituting these into (21) and the time-derivative of $V$ has new upper bound as follows:

$$
\dot{V}(x(t)) \leq \xi^{T}(t) \Phi \xi(t)
$$

where

$$
\xi^{T}(t)=\left[x^{T}(t) x^{T}\left(t-\frac{h}{2}\right) x^{T}(t-h) x^{T}\left(t-\frac{\tau}{2}\right) x^{T}(t-\tau) \int_{t-h}^{t} x^{T}(s) d s \int_{t-\tau}^{t} x^{T}(s) d s \quad D z(K x)\right] .
$$

$\Phi$ is defined as stated in (16).

If linear matrix inequality (16) is feasible, then we can get $\dot{V}(x(t))<0, \forall x \in \mathcal{S}$. Therefore, if there exist scalars $\alpha_{1}$ and $\alpha_{2}$ and a positive definite matrix $M$ satisfying (12) and there exist $P>0, Q=\left[Q_{i j}\right]_{2 \times 2}, Q_{i j}>0, R=\left[R_{i j}\right]_{2 \times 2}, R_{i j}>0, T_{i}>0,(i=$ $1,2,3,4,5,6), \widetilde{T}_{i}>0,(i=3,4)$, a matrix $\widehat{G}$, and a diagonal matrix $\Lambda$ of appropriate dimensions satisfying (16), then we can draw the neutral system which can be described by (1) and (3) is asymptotic stability. This completes the proof.

Remark 9. Theorem 8 gives a delay-dependent stability criterion for neutral system satisfying (1) and (3) by employing model transformation and delay-dividing approach. Model transformation may be an old technique and can be seen in [7]. But a new stability condition on the operator $\mathscr{L}$ is adopted in this paper to reduce the conservatism of the neutral system. Moreover, the proposed stability condition is not only on the discrete delays but also on the neutral delays, while the conditions in $[31,32]$ depend only on consideration of the discrete delays.

Remark 10. By dividing the neutral delay interval $[-\tau, 0]$ into $[-\tau,-\tau / 2]$ and $[-\tau / 2,0]$, we choose different functional on $[-\tau,-\tau / 2]$ and $[-\tau / 2,0]$, respectively. The discrete delay $h$ is to take the same approach. Then the division provides extra freedom for delay terms and reduces the conservation.

We can easily get a delay-dependent stability criterion for system (1) with $\tau \equiv h$. This paper will show the asymptotic stability criterion for this case in Corollary 11.

Corollary 11. Given scalars $\alpha_{1}$ and $\alpha_{2}$, a matrix $G$, and a diagonal matrix $\Lambda$ of appropriate dimensions, the neutral systems (1) and (3) when $\tau \equiv h$ is asymptotically stable if there exists a positive definite matrix $M$ satisfying (12) and there exist $P>0, Q=\left[Q_{i j}\right]_{2 \times 2}, Q_{i j}>0, T_{i}>0,(i=1,2,3), \widetilde{T_{2}}>0$ such that the following symmetric linear matrix inequality holds:

$$
\bar{\Phi}=\left[\begin{array}{ccccc}
\bar{\Phi}_{11} & \bar{\Phi}_{12} & \bar{\Phi}_{13} & \bar{\Phi}_{14} & \bar{\Phi}_{15} \\
\star & \bar{\Phi}_{22} & \bar{\Phi}_{23} & 0 & 0 \\
\star & \star & \bar{\Phi}_{33} & 0 & \bar{\Phi}_{35} \\
\star & \star & \star & \bar{\Phi}_{44} & \bar{\Phi}_{45} \\
\star & \star & \star & \star & \Phi_{55}
\end{array}\right]<0
$$

$$
\begin{aligned}
\text { where } \widehat{G} & =\Lambda G, \widetilde{T_{2}}=T_{2} T_{3}^{-1} T_{2} \\
\bar{\Phi}_{11} & =\widehat{A}_{c}^{T} P+P \widehat{A}_{c}+Q_{11}+T_{1}+\frac{h^{2}}{2}\left(\widetilde{T_{2}}+T_{3}\right), \\
\bar{\Phi}_{22} & =Q_{22}-Q_{11}, \\
\bar{\Phi}_{33} & =-Q_{22}-T_{1}, \\
\bar{\Phi}_{44} & =-T_{2}, \\
\bar{\Phi}_{55} & =-2 \Lambda, \\
\bar{\Phi}_{12} & =Q_{12}, \\
\bar{\Phi}_{13} & =-\widehat{A}_{c}^{T} P D \\
\bar{\Phi}_{14} & =\widehat{A}_{c}^{T} P B \\
\bar{\Phi}_{15} & =-P C+\widehat{G}, \\
\bar{\Phi}_{23} & =-Q_{12}, \\
\bar{\Phi}_{35} & =D^{T} P C \\
\bar{\Phi}_{45} & =-B^{T} P C .
\end{aligned}
$$

Proof. Define a legitimate Lyapunov functional candidate as

$$
\begin{aligned}
V(x(t))= & V_{1}(x(t))+V_{2}(x(t))+V_{3}(x(t)) \\
& +V_{4}(x(t))+V_{5}(x(t)),
\end{aligned}
$$

where

$$
\begin{aligned}
V_{1}(x(t))=\mathscr{L}^{T}(x(t)) P \mathscr{L}(x(t)) \\
V_{2}(x(t))=\int_{t-(h / 2)}^{t}\left[x\left(s-\frac{h}{2}\right)\right]^{T} \\
\quad \times\left[\begin{array}{cc}
Q_{11} & Q_{12} \\
\star & Q_{22}
\end{array}\right]\left[x\left(s-\frac{h}{2}\right)\right] d s,
\end{aligned}
$$




$$
\begin{aligned}
& V_{3}(x(t))=\int_{t-h}^{t} x^{T}(s) T_{1} x(s) d s \\
& V_{4}(x(t))=\int_{t-h}^{t}\left[\int_{s}^{t} x^{T}\left(s_{1}\right) d s_{1}\right] T_{2}\left[\int_{s}^{t} x\left(s_{2}\right) d s_{2}\right] d s \\
& V_{5}(x(t))=\frac{1}{2} \int_{t-h}^{t}(s-t+h)^{2} x^{T}(s) T_{3} x(s) d s
\end{aligned}
$$

where $P>0, Q=\left[Q_{i j}\right]_{2 \times 2}, Q_{i j}>0$ and $T_{i}>0, i=1,2,3$.

According to (30), we obtain

$$
\begin{aligned}
& \dot{V}_{1}(x(t))=x^{T}\left[\widehat{A}_{c}^{T} P+P \widehat{A}_{c}\right] x+2 x^{T}(t-h) D^{T} P C D z(K x) \\
& +2 x^{T}(t) \widehat{A}_{c}^{T} P B\left[\int_{t-h}^{t} x(s) d s\right] \\
& -2 x^{T} P C D z(K x)-2 x^{T}(t) \widehat{A}_{c}^{T} P D x(t-h) \\
& -2\left[\int_{t-h}^{t} x^{T}(s) d s\right] B^{T} P C D z(K x), \\
& \dot{V}_{2}(x(t))=\left[\begin{array}{c}
x(t) \\
x\left(t-\frac{h}{2}\right)
\end{array}\right]^{T}\left[\begin{array}{cc}
Q_{11} & Q_{12} \\
\star & Q_{22}
\end{array}\right]\left[\begin{array}{c}
x(t) \\
x\left(t-\frac{h}{2}\right)
\end{array}\right] \\
& -\left[\begin{array}{c}
x\left(t-\frac{h}{2}\right) \\
x(t-h)
\end{array}\right]^{T}\left[\begin{array}{cc}
Q_{11} & Q_{12} \\
\star & Q_{22}
\end{array}\right]\left[\begin{array}{c}
x\left(t-\frac{h}{2}\right) \\
x(t-h)
\end{array}\right], \\
& \dot{V}_{3}(x(t))=x^{T}(t) T_{1} x(t)-x^{T}(t-h) T_{1} x(t-h), \\
& \dot{V}_{4}(x(t)) \leq-\left[\int_{t-h}^{t} x^{T}\left(s_{1}\right) d s_{1}\right] T_{2}\left[\int_{t-h}^{t} x\left(s_{2}\right) d s_{2}\right] \\
& +\frac{h^{2}}{2} x^{T}(t) \widetilde{T_{2}} x(t) \\
& +\int_{t-h}^{t}(s-t+h) x^{T}(s) T_{3} x(s) d s
\end{aligned}
$$

where $\widetilde{T_{2}}>0$, since $\widetilde{T_{2}}=T_{2} T_{3}^{-1} T_{2}$. Considering

$$
\begin{aligned}
\dot{V}_{5}(x(t))= & \frac{1}{2} h^{2} x^{T}(t) T_{3} x(t) \\
& -\int_{t-h}^{t}(s-t+h) x^{T}(s) T_{3} x(s) d s
\end{aligned}
$$

given that $x(t) \in \mathcal{S}$, which has been discussed in [21]. From Lemma 7, the time-derivative of $V$ has new upper bound as follows:

$$
\begin{aligned}
\dot{V}(x(t))= & \dot{V}_{1}(x(t))+\dot{V}_{2}(x(t)) \\
& +\dot{V}_{3}(x(t))+\dot{V}_{4}(x(t))+\dot{V}_{5}(x(t)) \\
& -2 D z^{T}(K x) \Lambda[D z(K x)-G x] \\
\leq & \bar{\xi}^{T}(t) \bar{\Phi} \bar{\xi}(t),
\end{aligned}
$$

where $\bar{\Phi}$ is defined as stated in (27) and

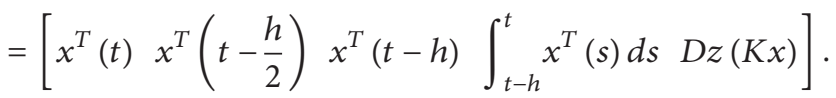

Therefore, if there exist scalars $\alpha_{1}$ and $\alpha_{2}$ and a positive definite matrix $M$ satisfying (12) and there exist $P>0$, $\widehat{P}>0, Q=\left[Q_{i j}\right]_{2 \times 2}, Q_{i j}>0, T_{i}>0,(i=1,2,3)$, $\widetilde{T_{2}}>0$ satisfying (27), then we can draw the identical neutral delay and discrete delay system is asymptotic stability. This completes the proof.

Furthermore, since the model transformation which usually may lead to some degree of conservatism of the method, we establish the following theorem based on Theorem 8 instead of using the model transformation.

Theorem 12. Given a matrix $G$ and a diagonal matrix $\Lambda$ of appropriate dimensions, the neutral system with time-delay and actuator saturation which can be described by (1) and (3) is asymptotically stable if $\|D\|<1$ and there exist $P>0$, $Q=\left[Q_{i j}\right]_{2 \times 2}, Q_{i j}>0, R=\left[R_{i j}\right]_{2 \times 2}, R_{i j}>0, T_{i}>0,(i=$ $1,2,3,4,5,6), \widetilde{T}_{i}>0,(i=3,4)$ such that the following symmetric linear matrix inequality holds:

$$
\Phi=\left[\begin{array}{cccccccc}
\Phi_{11} & \Phi_{12} & \Phi_{13} & \Phi_{14} & \Phi_{15} & 0 & 0 & \Phi_{18} \\
\star & \Phi_{22} & \Phi_{23} & 0 & 0 & 0 & 0 & 0 \\
\star & \star & \Phi_{33} & 0 & \Phi_{35} & 0 & 0 & 0 \\
\star & \star & \star & \Phi_{44} & \Phi_{45} & 0 & 0 & 0 \\
\star & \star & \star & \star & \Phi_{55} & 0 & 0 & \Phi_{58} \\
\star & \star & \star & \star & \star & \Phi_{66} & 0 & 0 \\
\star & \star & \star & \star & \star & \star & \Phi_{77} & 0 \\
\star & \star & \star & \star & \star & \star & \star & \Phi_{88}
\end{array}\right]<0,
$$

where $\Phi_{13}=P B, \Phi_{35}=-B^{T} P D$,

$$
\begin{aligned}
\Phi_{11}= & A_{c}^{T} P+P A_{c}+Q_{11}+R_{11}+T_{1}+T_{2} \\
& +\frac{h^{2}}{2}\left(\widetilde{T_{3}}+T_{5}\right)+\frac{\tau^{2}}{2}\left(\widetilde{T_{4}}+T_{6}\right)
\end{aligned}
$$

and other notations are identical with Theorem 8.

Proof. We replace $\mathscr{L}(x(t))$ with $\mathscr{D}(x(t))$ in $V_{1}(x(t))$, and then follow the same procedure to prove Theorem 8 . Thus it will complete the proof.

Remark 13. It should be pointed out that Theorem 12 is less conservative than Theorem 8 , which can be verified by the numerical example. However, if the condition $\|D\|<1$ cannot be satisfied while (12) can be satisfied, Theorem 12 is no longer applicable and we may apply Theorem 8 in this paper. 
3.2. Asymptotic Stability for Uncertain Neutral System. Furthermore, let us consider the following uncertain neutral system with time-delay and actuator saturation:

$$
\begin{aligned}
\dot{x}(t)-D \dot{x}(t-\tau)= & (A+\Delta A(t)) x(t)+B x(t-h) \\
& +(C+\Delta C(t)) \text { Sat }(u(t)),
\end{aligned}
$$

where $\Delta A(t)$ and $\Delta C(t)$ stand for the uncertainties. For simplicity, The constant parameter matrices $A, B, C$, and $D$ are square matrices. The uncertainties are not known but the spectral norm bound is known and can be described as

$$
\|\Delta A(t)\| \leq \alpha, \quad\|\Delta C(t)\| \leq \beta, \quad \forall t \geq 0 .
$$

Utilizing the nonlinear function $D z(\cdot)$, we can also rewrite the uncertain neutral system in the following form:

$$
\begin{aligned}
\dot{x}(t)-D \dot{x}(t-\tau)= & \left(A_{c}+\Delta A(t)-\Delta C(t) K\right) x(t) \\
& +B x(t-h)+(C+\Delta C(t)) D z(K x(t)),
\end{aligned}
$$

where $A_{c}$ is defined as the same with certain neutral system with time-delay and actuator saturation. Specially, when $\|\Delta A(t)\|=0$ and $\|\Delta C(t)\|=0$ the uncertain neutral system becomes the certain case.

Similarly, we employ the operator $\mathscr{L}: C_{0} \rightarrow \mathbb{R}^{n}$ with

$$
\mathscr{L}(x(t))=x(t)+B \int_{t-h}^{t} x(s) d s-D x(t-\tau) .
$$

Then we obtain the transformed system

$$
\frac{d}{d t} \mathscr{L}(x(t))=\overline{A_{c}} x(t)+(C+\Delta C(t)) D z(K x(t)),
$$

where $\overline{A_{c}}=\widehat{A}_{c}+\Delta A(t)-\Delta C(t) K=A+B+C K+\Delta A(t)-$ $\Delta C(t) K$.

With regard to the uncertain neutral system, we can also prove the following theorem.

Theorem 14. Given scalars $\alpha_{1}$ and $\alpha_{2}, a$ matrix $G$, and $a$ diagonal matrix $\Lambda$ of appropriate dimensions, the uncertain neutral system which can be described as (37) with feedback control (3) is asymptotically stable if there exists a positive definite matrix $M$ satisfying (12) and there exist $P>0$, $Q=\left[Q_{i j}\right]_{2 \times 2}, Q_{i j}>0, R=\left[R_{i j}\right]_{2 \times 2}, R_{i j}>0, T_{i}>0$, ( $i=1,2,3,4,5,6), \widetilde{T}_{i}>0,(i=3,4)$ such that the following symmetric linear matrix inequality holds:

$$
\Xi=\left[\begin{array}{cccccccc}
\Xi_{11} & \Xi_{12} & 0 & \Xi_{14} & \Xi_{15} & \Xi_{16} & 0 & \Xi_{18} \\
\star & \Xi_{22} & \Xi_{23} & 0 & 0 & 0 & 0 & 0 \\
\star & \star & \Xi_{33} & 0 & 0 & 0 & 0 & 0 \\
\star & \star & \star & \Xi_{44} & \Xi_{45} & 0 & 0 & 0 \\
\star & \star & \star & \star & \Xi_{55} & 0 & 0 & 0 \\
\star & \star & \star & \star & \star & \Xi_{66} & 0 & 0 \\
\star & \star & \star & \star & \star & \star & \Xi_{77} & 0 \\
\star & \star & \star & \star & \star & \star & \star & \Xi_{88}
\end{array}\right]<0,
$$

where $\widehat{G}=\Lambda G, \widetilde{T_{3}}=T_{3} T_{5}^{-1} T_{3}$, and $\widetilde{T_{4}}=T_{4} T_{6}^{-1} T_{4}$

$$
\begin{aligned}
& \Xi_{11}=\widehat{A}_{c}^{T} P+P \widehat{A}_{c}+\frac{1}{\gamma_{1}} \widehat{P}+\frac{1}{\gamma_{2}} \widehat{P} \\
& +\left(\gamma_{1}+\gamma_{3}+\gamma_{5}\right)\left(\alpha^{2}+2 \alpha \beta\|K\|+\beta^{2}\|K\|^{2}\right) I \\
& +Q_{11}+R_{11}+T_{1}+T_{2}+\frac{h^{2}}{2}\left(\widetilde{T_{3}}+T_{5}\right)+\frac{\tau^{2}}{2}\left(\widetilde{T_{4}}+T_{6}\right), \\
& \Xi_{22}=Q_{22}-Q_{11} \\
& \Xi_{33}=-Q_{22}-T_{1} \text {, } \\
& \Xi_{44}=R_{22}-R_{11} \text {, } \\
& \Xi_{55}=\left(\frac{1}{\gamma_{3}}+\frac{1}{\gamma_{4}}\right) D^{T} \widehat{P} D-R_{22}-T_{2}, \\
& \Xi_{66}=\left(\frac{1}{\gamma_{5}}+\frac{1}{\gamma_{6}}\right) B^{T} \widehat{P} B-T_{3}, \\
& \Xi_{77}=-T_{4} \\
& \Xi_{88}=\left(\gamma_{2}+\gamma_{4}+\gamma_{6}\right)\left(\beta^{2}+2 \beta\|C\|+\|C\|^{2}\right) I-2 \Lambda, \\
& \Xi_{12}=Q_{12} \text {, } \\
& \Xi_{14}=R_{12} \text {, } \\
& \Xi_{15}=-\widehat{A}_{c}^{T} P D, \\
& \Xi_{16}=\widehat{A}_{c}^{T} P B, \\
& \Xi_{18}=\widehat{G}, \\
& \Xi_{23}=-Q_{12} \text {, } \\
& \Xi_{45}=-R_{12} \text {. }
\end{aligned}
$$

Proof. In the same way, we define a legitimate Lyapunov functional candidate as

$$
\begin{aligned}
V(x(t))= & V_{1}(x(t))+V_{2}(x(t))+V_{3}(x(t)) \\
& +V_{4}(x(t))+V_{5}(x(t))+V_{6}(x(t)),
\end{aligned}
$$

where $V_{1}(x(t)), V_{2}(x(t)), V_{3}(x(t)), V_{4}(x(t)), V_{5}(x(t)), V_{6}(x(t))$ are the same with those in Theorem 8. Given that $x(t) \in \mathcal{S}$, which has been discussed in [21]. From Lemma 7, it also follows that

$$
\begin{aligned}
\dot{V}(x(t)) \leq & \dot{V}_{1}(x(t))+\dot{V}_{2}(x(t))+\dot{V}_{3}(x(t)) \\
& +\dot{V}_{4}(x(t))+\dot{V}_{5}(x(t))+\dot{V}_{6}(x(t)) \\
& -2 D z^{T}(K x) \Lambda[D z(K x)-G x] .
\end{aligned}
$$


In fact

$$
\begin{aligned}
& \dot{V}_{1}(x(t))=2\left\{x^{T}(t)-x^{T}(t-\tau) D^{T}\right. \\
& \left.+\left[\int_{t-h}^{t} x^{T}(s) d s\right] B^{T}\right\} \\
& \times P\left[\overline{A_{c}} x(t)+(C+\Delta C) D z(K x)\right] \\
& =2 x^{T} P \overline{A_{c}} x+2 x^{T} P(C+\Delta C) D z(K x) \\
& -2 x^{T}{\overline{A_{c}}}^{T} P D x(t-\tau)-2 x^{T}(t-\tau) D^{T} \\
& \times P(C+\Delta C) D z(K x) \\
& +2 x^{T}(t){\overline{A_{c}}}^{T} P B\left[\int_{t-h}^{t} x(s) d s\right] \\
& +2\left[\int_{t-h}^{t} x^{T}(s) d s\right] B^{T} P(C+\Delta C) D z(K x) \\
& =2 x^{T} P \widehat{A}_{c} x+2 x^{T} P(\Delta A-\Delta C K) x \\
& +2 x^{T} P(C+\Delta C) D z(K x) \\
& -2 x^{T}(t-\tau) D^{T} P \widehat{A}_{c} x(t) \\
& -2 x^{T}(t-\tau) D^{T} P(\Delta A-\Delta C K) x \\
& -2 x^{T}(t-\tau) D^{T} P(C+\Delta C) D z(K x) \\
& +2\left[\int_{t-h}^{t} x^{T}(s) d s\right] B^{T} P \widehat{A}_{c} x \\
& +2\left[\int_{t-h}^{t} x^{T}(s) d s\right] B^{T} P(\Delta A-\Delta C K) x \\
& +2\left[\int_{t-h}^{t} x^{T}(s) d s\right] B^{T} P(C+\Delta C) D z(K x) \\
& \leq x^{T}\left[\widehat{A}_{c}^{T} P+P \widehat{A}_{c}\right] x-2 x^{T}(t) \widehat{A}_{c}^{T} P D x(t-\tau) \\
& +2 x^{T}(t) \widehat{A}_{c}^{T} P B\left[\int_{t-h}^{t} x(s) d s\right] \\
& +\frac{1}{\gamma_{1}} x^{T} \widehat{P} x+\gamma_{1} x^{T}(\Delta A-\Delta C K)^{T} \\
& \times(\Delta A-\Delta C K) x \\
& +\frac{1}{\gamma_{2}} x^{T} \widehat{P} x+\gamma_{2} D z^{T}(K x)(C+\Delta C)^{T} \\
& \times(C+\Delta C) D z(K x) \\
& +\frac{1}{\gamma_{3}} x^{T}(t-\tau) D^{T} \widehat{P} D x(t-\tau)
\end{aligned}
$$

$$
\begin{aligned}
& +\gamma_{3} x^{T}(\Delta A-\Delta C K)^{T}(\Delta A-\Delta C K) x \\
& +\frac{1}{\gamma_{4}} x^{T}(t-\tau) D^{T} \widehat{P} D x(t-\tau) \\
& +\gamma_{4} D z^{T}(K x)(C+\Delta C)^{T}(C+\Delta C) D z(K x) \\
& +\frac{1}{\gamma_{5}}\left[\int_{t-h}^{t} x^{T}(s) d s\right] B^{T} \widehat{P} B\left[\int_{t-h}^{t} x(s) d s\right] \\
& +\gamma_{5} x^{T}(\Delta A-\Delta C K)^{T}(\Delta A-\Delta C K) x \\
& +\frac{1}{\gamma_{6}}\left[\int_{t-h}^{t} x^{T}(s) d s\right] B^{T} \widehat{P} B\left[\int_{t-h}^{t} x(s) d s\right] \\
& +\gamma_{6} D z^{T}(K x)(C+\Delta C)^{T}(C+\Delta C) D z(K x) \\
& +x^{T}\left\{\left(\widehat{A}_{c}^{T} P+P \widehat{A}\right)+\left(\frac{1}{\gamma_{1}}+\frac{1}{\gamma_{2}}\right) \widehat{P}\right. \\
& + \\
& \left.\left.+\int_{t-h}^{t} x \widehat{x}^{T}(t) \widehat{A}_{c}^{T} P D x(t-\tau) d s\right] \widehat{A}_{c}^{T} P B\right] \\
& +\left(\gamma_{2}+\gamma_{4}+\gamma_{6}\right)\left(\beta^{2}+2 \beta\|C\|+\|C\|^{2}\right) \\
& +\left(\gamma_{1}+\gamma_{3}+\gamma_{5}\right) \\
& \left.+\times\left(\alpha^{2}+2 \alpha \beta\|K\|+\beta^{2}\|K\|^{2}\right) I\right\} x
\end{aligned}
$$

where $\widehat{P}>0$, since $\widehat{P}=P^{2}$.

While $\dot{V}_{2}(x(t)), \dot{V}_{3}(x(t)), \dot{V}_{4}(x(t)), \dot{V}_{5}(x(t))$, and $\dot{V}_{6}(x(t))$ are obtained by the same method in Theorem 8 . Then substituting these into (45), the time-derivative of $V$ has new upper bound as follows:

$$
\begin{aligned}
\dot{V}(x(t)) & \leq \dot{V}(x(t))-2 D z^{T}(K x) \Lambda[D z(K x)-G x] \\
& \leq \xi^{T}(t) \Xi \xi(t),
\end{aligned}
$$

where $x(t) \in \mathcal{S}$, which has been discussed in [21], and 


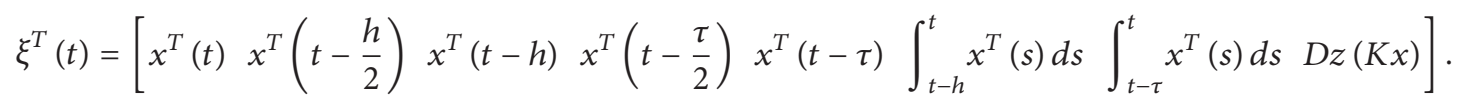

$\Xi$ is defined as stated in (42).

If linear matrix inequality (42) is feasible, then we can also get $\dot{V}(x(t))<0, \forall x \in \mathcal{S}$. Therefore, if there exist scalars $\alpha_{1}$ and $\alpha_{2}$ and a positive definite matrix $M$ satisfying (12) and there exist $P>0, \widehat{P}>0, Q=\left[Q_{i j}\right]_{2 \times 2}, Q_{i j}>0, R=\left[R_{i j}\right]_{2 \times 2}$, $R_{i j}>0, T_{i}>0,(i=1,2,3,4,5,6), \widetilde{T}_{i}>0,(i=3,4)$ satisfying (42) for real scalars $\gamma_{i}>0,(i=1,2,3,4,5,6)$, then we can draw the uncertain neutral system which can be described by (37) and (3) is asymptotic stability. This completes the proof.

Theorem 14 provides a new asymptotic stability condition for a class of uncertain neutral system which can be described as (37) and (3). We can also get a Corollary 15 which is a special case of Theorem 14 .

Corollary 15. Given scalars $\alpha_{1}$ and $\alpha_{2}$, a matrix $G$, and a diagonal matrix $\Lambda$ of appropriate dimensions, the uncertain neutral system (37) with feedback control (3) when $\tau \equiv h$ is asymptotically stable if there exists a positive definite matrix $M$ satisfying (12) and there exist $P>0, Q=\left[Q_{i j}\right]_{2 \times 2}, Q_{i j}>0$, $T_{i}>0,(i=1,2,3), \widetilde{T_{2}}>0$ such that the following symmetric linear matrix inequality holds:

$$
\bar{\Xi}=\left[\begin{array}{ccccc}
\bar{\Xi}_{11} & \bar{\Xi}_{12} & \bar{\Xi}_{13} & \bar{\Xi}_{14} & \bar{\Xi}_{15} \\
\star & \bar{\Xi}_{22} & \bar{\Xi}_{23} & 0 & 0 \\
\star & \star & \bar{\Xi}_{33} & 0 & 0 \\
\star & \star & \star & \bar{\Xi}_{44} & 0 \\
\star & \star & \star & \star & \bar{\Xi}_{55}
\end{array}\right]<0
$$

where $\widehat{G}=\Lambda G, \widetilde{T_{2}}=T_{2} T_{3}^{-1} T_{2}$

$$
\begin{aligned}
\bar{\Xi}_{11}= & \widehat{A}_{c}^{T} P+P \widehat{A}_{c}+\frac{1}{\gamma_{1}} \widehat{P}+\frac{1}{\gamma_{2}} \widehat{P} \\
& +\left(\gamma_{1}+\gamma_{3}+\gamma_{5}\right)\left(\alpha^{2}+2 \alpha \beta\|K\|+\beta^{2}\|K\|^{2}\right) I \\
& +Q_{11}+T_{1}+\frac{h^{2}}{2}\left(\widetilde{T_{2}}+T_{3}\right), \\
\bar{\Xi}_{22}= & Q_{22}-Q_{11}, \\
\bar{\Xi}_{33}= & \left(\frac{1}{\gamma_{3}}+\frac{1}{\gamma_{4}}\right) D^{T} \widehat{P} D-Q_{22}-T_{1}, \\
\bar{\Xi}_{44}= & \left(\frac{1}{\gamma_{5}}+\frac{1}{\gamma_{6}}\right) B^{T} \widehat{P} B-T_{2}, \\
\bar{\Xi}_{55}= & \left(\gamma_{2}+\gamma_{4}+\gamma_{6}\right)\left(\beta^{2}+2 \beta\|C\|+\|C\|^{2}\right) I-2 \Lambda, \\
\bar{\Xi}_{12}= & Q_{12}, \\
\bar{\Xi}_{13}= & -\widehat{A}_{c}^{T} P D,
\end{aligned}
$$

where $\Xi_{13}=P B, \Xi_{35}=-B^{T} P D$,

$$
\begin{aligned}
\Xi_{11}= & A_{c}^{T} P+P A_{c}+\left(\frac{1}{\gamma_{1}}+\frac{1}{\gamma_{2}}\right) \widehat{P} \\
& +\left(\gamma_{1}+\gamma_{3}\right)\left(\alpha^{2}+2 \alpha \beta\|K\|+\beta^{2}\|K\|^{2}\right) I+Q_{11} \\
& +R_{11}+T_{1}+T_{2}+\frac{h^{2}}{2}\left(\widetilde{T_{3}}+T_{5}\right)+\frac{\tau^{2}}{2}\left(\widetilde{T_{4}}+T_{6}\right) \Xi_{66} \\
= & -T_{3}, \Xi_{88}=\left(\gamma_{2}+\gamma_{4}\right)\left(\beta^{2}+2 \beta\|C\|+\|C\|^{2}\right) I-2 \Lambda
\end{aligned}
$$

which are the same as (30).

We follow the same processes in Corollary 11 and Theorem 14, then the proof can be easily completed.

Similar to the certain system, we also give the following less conservative condition for the uncertain case.

Theorem 16. Given a matrix $G$ and a diagonal matrix $\Lambda$ of appropriate dimensions, the uncertain neutral system which can be described as (37) with feedback control (3) is asymptotically stable if $\|D\|<1$ and there exist $P>0$, $Q=\left[Q_{i j}\right]_{2 \times 2}, Q_{i j}>0, R=\left[R_{i j}\right]_{2 \times 2}, R_{i j}>0, T_{i}>0$, $(i=1,2,3,4,5,6), \widetilde{T}_{i}>0,(i=3,4)$ such that the following symmetric linear matrix inequality holds:

$$
\Xi=\left[\begin{array}{cccccccc}
\Xi_{11} & \Xi_{12} & \Xi_{13} & \Xi_{14} & \Xi_{15} & 0 & 0 & \Xi_{18} \\
\star & \Xi_{22} & \Xi_{23} & 0 & 0 & 0 & 0 & 0 \\
\star & \star & \Xi_{33} & 0 & \Xi_{35} & 0 & 0 & 0 \\
\star & \star & \star & \Xi_{44} & \Xi_{45} & 0 & 0 & 0 \\
\star & \star & \star & \star & \Xi_{55} & 0 & 0 & 0 \\
\star & \star & \star & \star & \star & \Xi_{66} & 0 & 0 \\
\star & \star & \star & \star & \star & \star & \Xi_{77} & 0 \\
\star & \star & \star & \star & \star & \star & \star & \Xi_{88}
\end{array}\right]<0,
$$

and other notations are identical with Theorem 14.

Proof. We replace $\mathscr{L}(x(t))$ with $\mathscr{D}(x(t))$ in $V_{1}(x(t))$, and then follow the same procedure to prove Theorem 14 . Thus it will complete the proof. 


\section{Numerical Examples}

In this section, we present some numerical examples to illustrate the effectiveness of the proposed theoretical results.

\subsection{No Uncertainty Case}

Example 1. Consider the system (1) without saturation described by the following:

$$
\begin{aligned}
\dot{x}(t) & -\left[\begin{array}{cc}
-0.2 & 0 \\
0.2 & -0.2
\end{array}\right] \dot{x}(t-h) \\
& =\left[\begin{array}{cc}
-0.9 & 0.2 \\
0 & -0.9
\end{array}\right] x(t)+\left[\begin{array}{ll}
-1.1 & -0.2 \\
-0.1 & -1.1
\end{array}\right] x(t-h) .
\end{aligned}
$$

By Corollary 11 and Theorem 12, the maximum upper bound of $h$, which satisfies the LMI (27) and (35), can be calculated by solving a quasiconvex optimization problem. This neutral system is considered by the proposed methods in $[8,18,21,22,28]$. The results on the maximum upper bound of $h$ are compared in Table 1 .

From Table 1, we know that the maximum upper bound of delay $h=0.7204$ by Corollary 11, and $h=0.7211$ by and Theorem 12, respectively, while the maximum upper bound of delay $h=0.6233$ for [18], $h=0.7000$ for [28], $h=0.5658$ for [8], $h=0.7135$ for [21], and $h=0.7048$ for [22]. It is found that the maximum upper bound in this paper is larger than those in $[8,18,21,22,28]$. So it can be demonstrated that the delay-dependent stability condition in this paper yields less conservative results than those previous ones. Additionally, it can be seen that Theorem 12 is less conservative.

Necessarily, let us check the stability of the operator $\mathscr{L}(x(t))$ given in (9) when $h=0.7204$. By solving inequality (12), we have

$$
\alpha_{1}=0.4137, \quad \alpha_{2}=0.3625, \quad M=\left[\begin{array}{ll}
0.6157 & 0.0096 \\
0.0096 & 0.6278
\end{array}\right]
$$

which guarantees the stability of the operator.

Example 2. Consider a time-delay neutral system with an actuator saturated at level \pm 1 described as follows:

$$
\begin{aligned}
\dot{x}(t) & -\left[\begin{array}{cc}
0.15 & 0 \\
0 & 0.15
\end{array}\right] \dot{x}(t-\tau) \\
= & {\left[\begin{array}{cc}
-2 & 0 \\
1 & -3
\end{array}\right] x(t)+\left[\begin{array}{cc}
-1 & 0 \\
-0.8 & -1
\end{array}\right] x(t-0.6) } \\
& +\left[\begin{array}{cc}
1 & 2 \\
-1 & 4
\end{array}\right] \text { Sat }(u(t)) .
\end{aligned}
$$

It is found that the nominal system (57) is unstable and it is intended to stabilize the controlled system and find the range of delay time $\tau$ by using a state feedback controller $K$ to guarantee that the above system is asymptotically stable. We need to verify the stability of the operator $\mathscr{L}: C_{0} \rightarrow \mathbb{R}^{n}$ with

$$
\mathscr{L}(x(t))=x(t)+B \int_{t-h}^{t} x(s) d s-D x(t-\tau) .
$$

By solving inequality (12), we obtain

$$
\alpha_{1}=0.3346, \quad \alpha_{2}=0.5728, \quad M=\left[\begin{array}{ll}
0.5364 & 0.0043 \\
0.0043 & 0.3968
\end{array}\right]
$$

which guarantees the stability of the operator in Example 2.

In this paper, we adopt the feedback control matrix

$$
K=\left[\begin{array}{ll}
0.2062 & -0.1304 \\
0.3755 & 0.4847
\end{array}\right]
$$

in [22]. By using the LMI toolbox in MATLAB, here the solutions of the LMIs given in Theorems 8 and 12 are found to be $\tau<0.7566$ and $\tau<0.7572$. So this example guarantees the asymptotic stabilization of system (57) for $0 \leq \tau \leq 0.7572$. When $D=0$, we obtain $0 \leq \tau \leq 0.7828$ while the result of Niculescu guaranteeing the robust stabilization of system (57) is $0 \leq \tau \leq 0.3819$ in [33]. Hence, our result gives a less conservative result than that obtained by delay-dependent stability in [33].

\subsection{Uncertainty Case}

Example 3. Consider the system (55) in uncertainty case

$$
\begin{aligned}
\dot{x}(t) & -\left[\begin{array}{cc}
-0.2 & 0 \\
0.2 & -0.2
\end{array}\right] \dot{x}(t-h) \\
& =(A+\Delta A(t)) x(t)+\left[\begin{array}{ll}
-1.1 & -0.2 \\
-0.1 & -1.1
\end{array}\right] x(t-h),
\end{aligned}
$$

where $A$ is identical with the system (55). The system (61) has the norm bounded uncertainty $\|\Delta A(t)\| \leq 0.01$. The stability of the operator has been verified in Example 1. Then, by Corollary 15 and Theorem 16, the maximum upper bound of $h$, which satisfies the LMI (49) and (53), can be calculated by solving a quasiconvex optimization problem. This neutral system is considered by the proposed methods in [18], and methods in $[8,21,22,28]$ are no longer applicable to the uncertain case. The results on the maximum upper bound of $h$ are compared in Table 2.

From Table 2, we know that the maximum upper bound of delay $h=0.6852$ in this paper by Theorem 16, while the maximum upper bound of delay $h=0.5937$ for [18]. So it also can be demonstrated that the delay-dependent stability condition in this paper yields less conservative results than some previous ones.

Example 4. Consider the system (57) in uncertainty case

$$
\begin{aligned}
\dot{x}(t)- & {\left[\begin{array}{cc}
0.15 & 0 \\
0 & 0.15
\end{array}\right] \dot{x}(t-\tau) } \\
= & (A+\Delta A(t)) x(t)+\left[\begin{array}{cc}
-1 & 0 \\
-0.8 & -1
\end{array}\right] x(t-0.6) \text { Sat }(u(t)), \\
& +(C+\Delta C(t)),
\end{aligned}
$$


TABLE 1: Maximum upper bound of $h$ with different methods.

\begin{tabular}{lccccccc}
\hline Methods & {$[8]$} & {$[18]$} & {$[28]$} & {$[22]$} & {$[21]$} & Corollary 11 & Theorem 12 \\
\hline$h$ & 0.5658 & 0.6233 & 0.7000 & 0.7048 & 0.7135 & 0.7204 & 0.7211 \\
\hline
\end{tabular}

TABLE 2: Maximum upper bound of $h$ with different methods.

\begin{tabular}{|c|c|c|c|c|c|c|}
\hline Methods [8] & {$[18]$} & {$[28]$} & [22] & [21] & Corollary 15 & Theorem 16 \\
\hline- & 0.5937 & - & - & - & 0.6849 & 0.6852 \\
\hline
\end{tabular}

where $A$ and $C$ are identical with the system (57). The stability of the operator has been verified in Example 2. The system (62) has the norm bounded uncertainties

$$
\|\Delta A(t)\| \leq 0.1, \quad\|\Delta C(t)\| \leq 0.1 .
$$

No doubt that this uncertainty will decrease the initial state bound that guarantees the asymptotic stability of the system (62). In this paper, we obtain $0 \leq \tau \leq 0.4369$ by Theorem 16 which guarantees the robust stabilization of system (62).

\section{Conclusions}

The asymptotic stability of neutral delay-differential systems with saturating actuators in terms of the LMI approach is developed in this paper. Applying the model transformation and the delay-dividing approach, a new augmented Lyapunov functional is constructed and the delay-dependent stability conditions are derived by some integral inequalities. Then the conditions are extended to the uncertain system, which has the norm bounded uncertainties. Examples have been provided to compare with recent papers, which show our results are more effective than those in earlier reports.

\section{Conflict of Interests}

The authors declare that there is no conflict of interests regarding the publication of this paper.

\section{Acknowledgments}

The authors would like to thank the editor and the anonymous reviewers for their constructive comments and suggestions to improve the quality of the paper. This work is partly supported by the Fundamental Research Funds for the Central Universities.

\section{References}

[1] Y. Kuang, Delay Differential Equations with Applications in Population Dynamics, Academic Press, San Diego, Calif, USA, 1993.

[2] V. B. Kolmanovskii and A. Myshkis, Applied Theory of Functional Differential Equations, Kluwer Academic, Dordrecht, The Netherlands, 1999.

[3] S.-I. Niculescu, Delay Effects on Stability: A Robust Control Approach, vol. 269 of Lecture Notes in Control and Information Sciences, Springer, London, UK, 2001.
[4] C.-H. Lien, "New stability criterion for a class of uncertain nonlinear neutral time-delay systems," International Journal of Systems Science, vol. 32, no. 2, pp. 215-219, 2001.

[5] C.-H. Lien, K.-W. Yu, and J.-G. Hsieh, "Stability conditions for a class of neutral systems with multiple time delays," Journal of Mathematical Analysis and Applications, vol. 245, no. 1, pp. 2027, 2000.

[6] D. Ivănescu, S.-I. Niculescu, L. Dugard, J.-M. Dion, and E. I. Verriest, "On delay-dependent stability for linear neutral systems," Automatica, vol. 39, no. 2, pp. 255-261, 2003.

[7] E. Fridman, "New Lyapunov-Krasovskii functionals for stability of linear retarded and neutral type systems," Systems \& Control Letters, vol. 43, no. 4, pp. 309-319, 2001.

[8] J. D. Chen, C.-H. Lien, K. K. Fan, and J. H. Chou, "Criteria for asymptotic stability of a class of neutral systems via a LMI approach," IEE Proceedings: Control Theory and Applications, vol. 148, no. 6, pp. 442-447, 2001.

[9] J.-H. Park, "A new delay-dependent criterion for neutral systems with multiple delays," Journal of Computational and Applied Mathematics, vol. 136, no. 1-2, pp. 177-184, 2001.

[10] J.-H. Park, "Stability criterion for neutral differential systems with mixed multiple time-varying delay arguments," Mathematics and Computers in Simulation, vol. 59, no. 5, pp. 401-412, 2002.

[11] J.-H. Park, "Convex optimization approach to dynamic output feedback control for delay differential systems of neutral type," Journal of Optimization Theory and Applications, vol. 127, no. 2, pp. 411-423, 2005.

[12] J.-H. Park and S. Won, "Asymptotic stability of neutral systems with multiple delays," Journal of Optimization Theory and Applications, vol. 103, no. 1, pp. 183-200, 1999.

[13] J.-H. Park and S. Won, "A note on stability of neutral delaydifferential systems," Journal of the Franklin Institute, vol. 336, no. 3, pp. 543-548, 1999.

[14] O. M. Kwon, M. J. Park, J. H. Park, S. M. Lee, and E. J. Cha, "New delay-partitioning approaches to stability criteria for uncertain neutral systems with time-varying delays," Journal of the Franklin Institute, vol. 349, no. 9, pp. 2799-2823, 2012.

[15] Q.-L. Han, "On delay-dependent stability for neutral delaydifferential systems," International Journal of Applied Mathematics and Computer Science, vol. 11, no. 4, pp. 965-976, 2001.

[16] Q.-L. Han, "Robust stability of uncertain delay-differential systems of neutral type," Automatica, vol. 38, no. 4, pp. 719-723, 2002.

[17] S.-I. Niculescu, "On delay-dependent stability under model transformations of some neutral linear systems," International Journal of Control, vol. 74, no. 6, pp. 609-617, 2001.

[18] A. Haurani, H. H. Michalska, and B. Boulet, "Delay-dependent robust stabilization of uncertain neutral systems with saturating actuators," in Proceedings of the American Control Conference, pp. 509-514, Denver, Colo, USA, June 2003.

[19] A. H. Glattfelder and W. Schaufelberger, "Stability analysis of single loop control systems with saturation and antiresetwindup circuits," IEEE Transactions on Automatic Control, vol. 28, no. 12, pp. 1074-1081, 2003. 
[20] J.-J. Yan, J.-S. Lin, and T.-L. Liao, "Robust dynamic compensator for a class of time delay systems containing saturating control input," Chaos, Solitons \& Fractals, vol. 31, no. 5, pp. 1223-1231, 2007.

[21] J. M. G. da Silva Jr., A. Seuret, E. Fridman, and J. P. Richard, "Stabilisation of neutral systems with saturating control inputs," International Journal of Systems Science, vol. 42, no. 7, pp. 10931103, 2011

[22] P.-L. Liu, "Stabilization criteria for neutral time delay systems with saturating actuators," Journal of the Franklin Institute, vol. 347, no. 8, pp. 1577-1588, 2010.

[23] P.-L. Liu and H.-L. Hung, "Stability of bilinear time-delay systems with saturating actuators," in Proceedings of the IEEE International Symposium on Industrial Electronics (ISIE '99), pp. 1082-1086, Bled, Slovenia, July 1999.

[24] J. K. Hale and S. M. V. Lunel, Introduction to FunctionalDifferential Equations, vol. 99 of Applied Mathematical Sciences, Springer, New York, NY, USA, 1993.

[25] S. A. Rodrìguez, J.-M. Dion, and L. Dugard, "Stability of neutral time delay systems: a survey of some results," in Advances in Automatic Control, vol. 754, pp. 315-335, Kluwer Academic, Boston, Mass, USA, 2004.

[26] V. B. Kolmanovskii and J.-P. Richard, "Stability of some linear systems with delays," IEEE Transactions on Automatic Control, vol. 44, no. 5, pp. 984-989, 1999.

[27] V. L. Kharitonov, "Robust stability analysis of time-delay systems: a survey," in Proceedings of the IFAC Conference on System Structure and Control, pp. 1-12, Nantes, France, 1998.

[28] D. Yue, S. Won, and O. Kwon, "Delay dependent stability of neutral systems with time delay: an LMI approach," IEE Proceedings: Control Theory and Applications, vol. 150, no. 1, pp. 23-27, 2003.

[29] H. B. Ji, Algebra Foundation of Control Theory, University of Science and Technology of China Press, Hefei, China, 2008.

[30] S. Tarbouriech, J. M. G. da Silva Jr., and G. Garcia, "Delaydependent anti-windup strategy for linear systems with saturating inputs and delayed outputs," International Journal of Robust and Nonlinear Control, vol. 14, no. 7, pp. 665-682, 2004.

[31] B. Yang, J. C. Wang, X. J. Pan, and C. Q. Zhong, "Delaydependent criteria for robust stability of linear neutral systems with time-varying delay and nonlinear perturbations," International Journal of Systems Science, vol. 38, no. 6, pp. 511-518, 2007.

[32] Y.-Y. Cao and J. Lam, "Computation of robust stability bounds for time-delay systems with nonlinear time-varying perturbations," International Journal of Systems Science, vol. 31, no. 3, pp. 359-365, 2000.

[33] S.-I. Niculescu, J.-M. Dion, and L. Dugard, "Robust stabilization for uncertain time-delay systems containing saturating actuators," IEEE Transactions on Automatic Control, vol. 41, no. 5, pp. 742-747, 1996. 


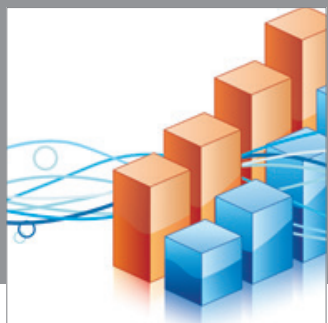

Advances in

Operations Research

mansans

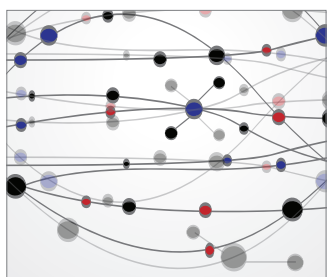

The Scientific World Journal
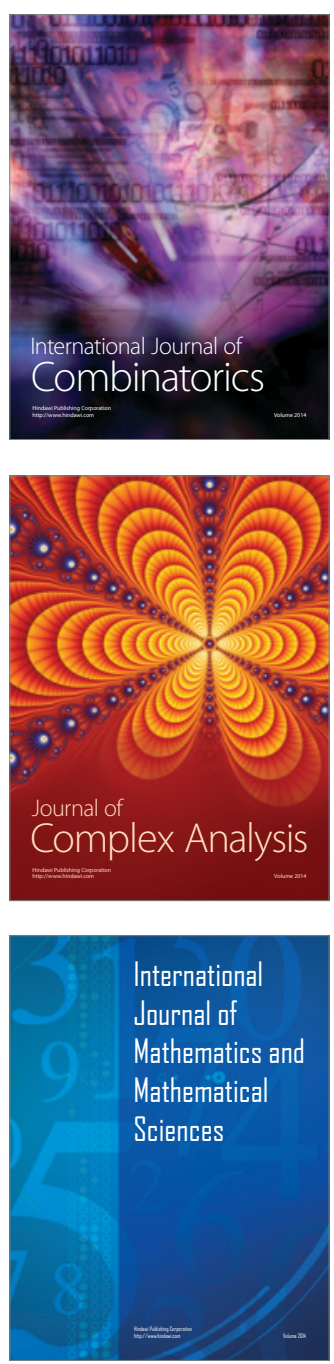
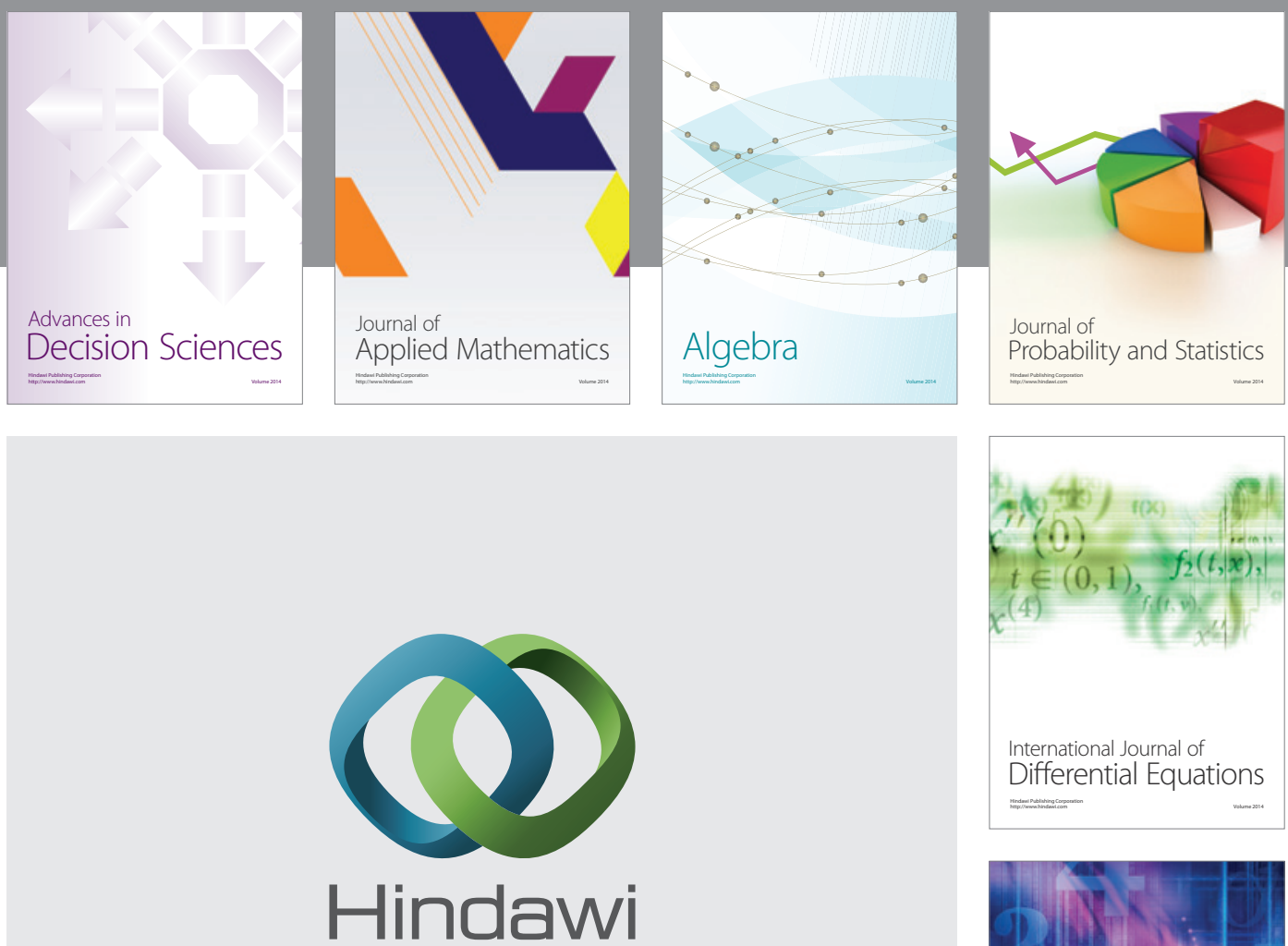

Submit your manuscripts at http://www.hindawi.com
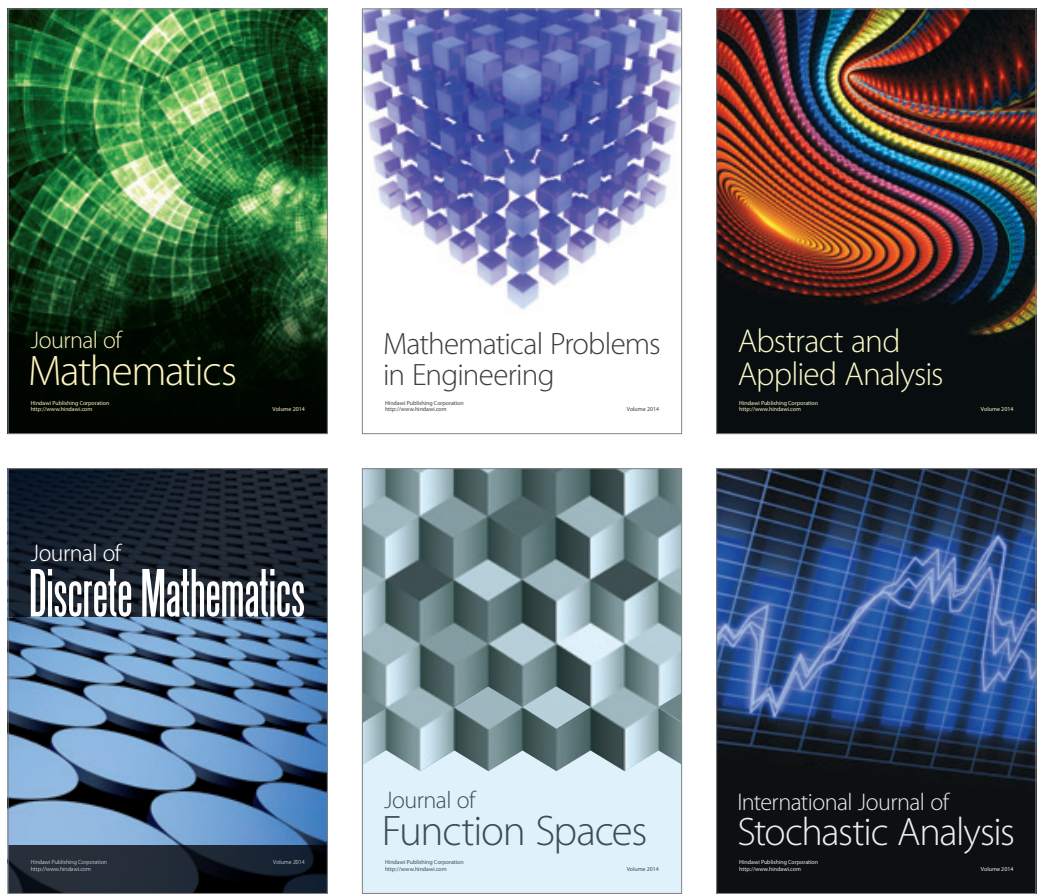

Journal of

Function Spaces

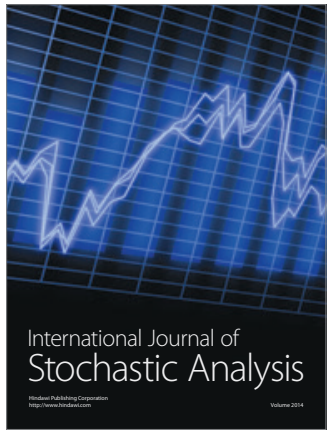

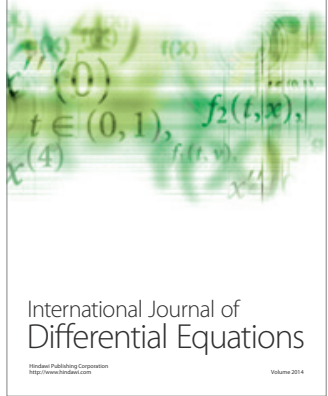
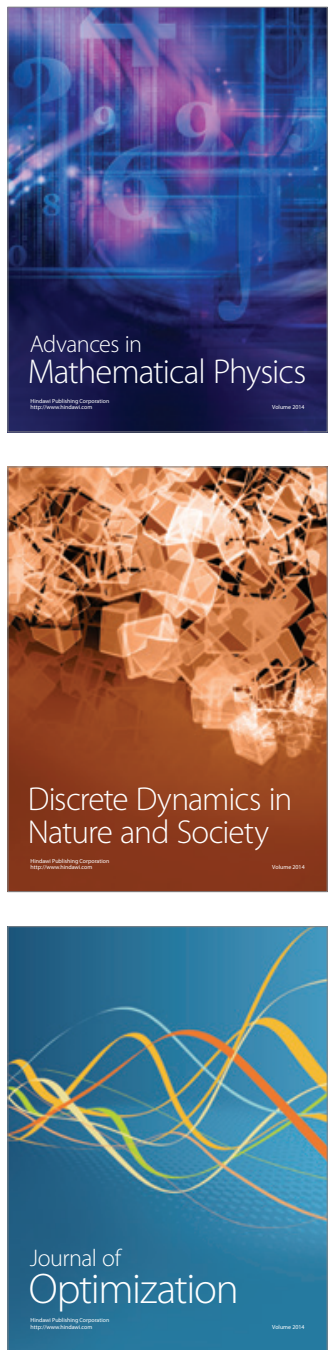